\title{
带鼓包的背负式大 $\mathbf{S}$ 弯进气道流场特性 及参数影响规律
}

\author{
贾洪印，周桂宇，唐静，吴晓军，马明生
}

(中国空气动力研究与发展中心 计算空气动力研究所, 四川 绵阳 621000)

\begin{abstract}
摘 要: 背负式进气口结合大 $S$ 弯内管道的进气系统设计方案, 由于其良好的隐身特性,在各类作战 飞机上得到了广泛应用。利用自主开发的大规模并行流场解算器, 对某带鼓包的背负式大 S 弯进气 道流场特性及参数影响规律进行了一体化数值模拟研究。首先介绍了采用的数值模拟方法,通过大 $\mathrm{S}$ 弯进气道模型, 验证了程序对进气道内分离流动预测的可靠性; 在此基础上,对带鼓包背负式大 $\mathrm{S}$ 弯进气道在超声速来流情况下入口激波分布、进气道内部流动情况以及出口流场的气流品质进行了 分析, 对比分析了不同鼓包高度和唇缘后掠角对进气道性能的影响, 结果显示: 进气道内独特的 $\mathrm{S}$ 弯 设计, 会导致流动分离和二次流,造成总压损失; 不同外部几何参数对进气道性能影响很大, 在一定范 围内,适当降低鼓包高度或减小唇缘后掠角,有利于进气道性能改善。
\end{abstract}

\section{关 键 词: 背负式; 大 $\mathrm{S}$ 弯进气道; 鼓包; 总压恢复;二次流; 参数影响}

\section{中图分类号: V211.3}

文献标志码: A

进气道作为推进系统的重要组成部分, 其性能 的优越将直接影响战斗机的性能。对于高隐身需求 的作战飞机,进气道系统的雷达散射面积能否有效 控制直接影响飞机的隐身性能 ${ }^{[1]}$ 。为降低飞机进 气系统的雷达散射面积, 进气道布局形式上可采用 背负式设计, 同时, 为实现对发动机进口端面的有效

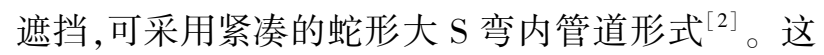
种高隐身的进气道系统设计近些年来受到了越来越 多飞行器设计者的青睐 ${ }^{[3]}$, 如美国的“B2”、“X-47” 等飞行器就采用了背负式进气道布局结合大 $\mathrm{S}$ 弯内 管道的设计方案。然而, 背负式进气道布局自身有 一定缺陷 ${ }^{[4]}$, 在较大迎角时不能很好利用高能来流 的冲压, 在设计时需充分考虑机身、机翼、进气道唇 口等部件对进气道性能的干扰影响。紧凑式大 $\mathrm{S}$ 弯 内管道设计容易造成流动分离, 使得总压恢复系数 降低, 流场畸变增大, 降低进气道性能 ${ }^{[5]}$ 。

在背负式进气道、无附面层隔板进气道( DSI) 研究方面, 罗普格鲁门公司的 Williams 等 ${ }^{[6]}$ 通过试 验方法对背负式进气道进行了大量研究, 指出背负
文章编号 : 1000-2758(2019)03-0572-08

式进气道的性能与飞机布局细节密切相关, 必须与 机体进行一体化综合设计。同时,通过对后掠的三 角翼外形的研究发现,边条漩浴对背部进气口的低 能量气流起到有利的 “扫除” 作用, 可以提高大迎角 时总压恢复和降低畸变。波音公司 Richard ${ }^{[7]}$ 对军 用飞机超声速进气道系统进行了详细介绍, 分析了 F35 采用的机身两侧 DSI 进气系统人口波系和鼓包 对附面层排除机理; 洛克希德马丁公司的 Hamstra 等 ${ }^{[8]}$, 采用先进的鼓包修型技术, 提升了 DSI 进气道 附面层排除能力 ; Kim 等 ${ }^{[9]}$ 将传统进气道压缩面置 换为三维鼓包, 通过优化鼓包长度和高度, 提升了进 气道的总压恢复, 降低了进气道出口畸变。Tan 和 Guo 等 ${ }^{[10]}$ 将背负式进气道方案与无附面层隔板技 术进行了结合, 研究了鼓包高度对 DSI 进气道性能 的影响。国内在背负式大 $\mathrm{S}$ 弯进气道设计及分离流 动控制方面也开展了大量的研究工作。南京航空航 天大学张航和谭慧俊等 ${ }^{[11]}$ 对安装在背部的类 X-47 狭缝式进气道进行了设计和仿真, 获得了该类进气 道的流动特征和工作特性。北京航空航天大学李大 
伟等 ${ }^{[12]}$ 针对无人机特点, 设计了一种短扩压、大偏 距、背负式 $\mathrm{S}$ 形进气道, 并通过仿真的方法, 分析了 进气道出口流场图谱和机身对进气道性能的影响。

在大 S 弯进气道性能预测方面, AIAA 从 2012 年至今共组织召开了 4 届推进空气动力学研讨 会 ${ }^{[13-15]}$, 针对设计的大 $S$ 弯进气道研究标模, 考核 现有 CFD 代码、网格和湍流模型在大 $\mathrm{S}$ 弯进气道内 分离流动预测的可靠性, 给出 CFD 模拟的指导原 则。来自 NASA、波音、DLR 等空气动力学研究机构 采用自主开发的 CFD 代码, 系统研究了不同网格、 不同湍流模型、不同来流速度对大 $\mathrm{S}$ 弯进气道内流 动的影响。

从目前公开发表的文献来看, 国内相关方面的 研究大多集中在对背负式进气道 ${ }^{[4]}$ 、DSI 进气 道 ${ }^{[16]}$ 、大 $\mathrm{S}$ 弯进气道流动特性和进气道内流动控制 技术的研究 ${ }^{[17]}$, 而针对背负式进气道布局、带大 $S$ 弯扩散段的 DSI 进气道一体化研究开展还相对 较少。

本文以带鼓包的背负式大 $\mathrm{S}$ 弯进气道为研究对 象, 采用非结构混合网格并结合自适应技术, 通过数 值求解 NS 方程, 对超声速来流条件下的进气道流 场特性及参数影响规律进行了一体化数值模拟研 究。通过 M2192 大 S 弯进气道模型, 验证了程序对 进气道内分离流动预测的可靠性。在此基础上, 对 带鼓包背负式大 S 弯进气道在超声速来流情况下人 口激波分布、进气道内部流动情况以及出口流场的 气流品质进行了分析, 重点考察了进气道人口鼓包 高度、唇缘后掠角等几何参数对进气道性能的影响, 得到了影响规律, 可以为我国类似带鼓包背负式大 $\mathrm{S}$ 弯进气道设计提供参考。

\section{1 数值方法}

本文研究采用中国空气动力研究与发展中心自 主开发的大规模并行流场解算器 MFlow 进行计算。 该解算器支持任意形状的网格单元, 方便对复杂流 场区域进行自适应加密, 变量存储于单元体心, 控制 方程采用有限体积方法进行离散, 时间离散采用隐 式的 LU-SGS 方法, 空间离散集成了目前国际主流 的空间离散格式和梯度求解方法, 湍流模拟可采用 SA 一方程和 SST 两方程湍流模型, 为了加速流场收 玫,软件采用了 FAS 融合的多重网格方法、局部时 间步长方法和基于 MPI 的大规模并行技术。目前,
该软件多次参加 AIAA 组织的阻力预测和高升力预 测会议, 数据对比中取得了较好的数值模拟 结果 ${ }^{[18-19]}$ 。

在本文的研究中,采用四面体和三棱柱单元混 合的非结构网格, 并结合了网格自适应技术, 对进气 道人口激波位置进行了精细捕捉。主控方程对流项 采用二阶迎风 Roe 通量差分裂格式进行离散, 时间 项采用隐式 LU-SGS 推进求解, 梯度求解采用节点 型 Gauss 方法, 限制器采用 Venkatakrishnan 限制器, 湍流模型采用 SA 一方程湍流模型和 SST 两方程湍 流模型。

\section{1 控制方程}

控制方程采用守恒形式的非定常可压缩 NS 方程

$$
\frac{\partial}{\partial t} \int_{\Omega} \boldsymbol{W} \mathrm{d} \Omega+\oint_{\partial \Omega}\left(\boldsymbol{F}_{c}-\boldsymbol{F}_{v}\right) \mathrm{d} S=0
$$

式中, $\Omega$ 表示控制体的体积, $\partial \Omega$ 表示控制体封闭面 的面积, $\boldsymbol{W}$ 为守恒变量, $\boldsymbol{F}_{c}$ 为无黏通量, $\boldsymbol{F}_{v}$ 为黏性 通量。

\section{2 网格自适应技术}

网格自适应技术的目的是使用尽量小的网格量 获得尽量高的计算精度, 基本的思想是增加局部区 域的网格分辨率, 以便更好地捕捉流场细节, 使计算 的精度增加。网格自适应技术常用于含有激波或漩 涡的流场, 可以得到更精细的激波和漩涡结构。自 适应探测器和网格细分策略是自适应技术的 2 个关 键技术。梯度探测器和法向马赫数探测器可以有效 地确定激波的位置, 摘增探测器可以确定浴核及附 近区域, 不同探测器的定义式如下:

法向马赫数探测器 ${ }^{[20]}$

$$
M_{n}=\frac{\boldsymbol{V}}{a} \cdot \frac{\nabla p}{|\nabla p|}
$$

熵增探测器 ${ }^{[21]}$

$$
\Delta s=s-s_{\infty}=\frac{R}{\gamma-1} \ln \frac{\frac{p}{p_{\infty}}}{\left(\frac{\rho}{\rho_{\infty}}\right)^{\gamma}}
$$

\section{3 湍流模型}

大 $\mathrm{S}$ 弯进气道内通常存在较强的逆压梯度和黏 性相互作用, 产生复杂的流动分离现象, 对湍流模型 要求较高。本文研究采用工程上应用广泛且相对成 熟的 2 个湍流模型, SA 一方程模型和 SST k- $\omega$ 两方 程湍流模型。 
将湍流方程写为如下统一的守恒积分形式

$$
\frac{\partial}{\partial t} \int_{\Omega} \boldsymbol{W}_{T} \mathrm{~d} \Omega+\oint_{\partial \Omega}\left(\boldsymbol{F}_{c, T}-\boldsymbol{F}_{v, T}\right) \mathrm{d} S=\int_{\Omega}\left(\boldsymbol{S}_{P}+\boldsymbol{S}_{D}\right) \mathrm{d} \Omega
$$

$\mathrm{SA}$ 一方程湍流模型公式的各部分及系数定义 参见文献 [22], SST k- $\omega$ 两方程湍流模型公式各部 分及系数定义参见文献 $[23]$ 。

本文中湍流模型方程的求解采用与主流控制方 程解耦的方式进行, 这样一方面可以减少对主流控 制方程的求解难度; 另一方面可以很好地利用已有 的层流流场解算器, 从而减少编程工作量; 最重要的 是可以减少计算量, 提高计算效率, 因为解耦避免了 求解高阶矩阵。

\section{2 方法验证}

为了验证本文采用的数值计算方法在进气道内 流计算中的可靠性, 我们选取了 RAE M2129 大 S 弯 进气道模型对程序进行了考核验证。该进气道模型 是在 NASA 和 UK Ministry of Defense 的支持下, 在 DRA/Bedford 13x9 英尺风洞进行了风洞试验, 并被 广泛应用于 CFD 程序验证与确认 ${ }^{[24]}$ 。

图 1 给出了该进气道外形的网格分布,计算采 用三棱柱和四面体混合的网格单元。网格单元总数 约为 617 万, 其中四面体单元 160 万, 三棱柱单元 457 万,附面层第一层间距按 $y+=1$ 选取,物面法向 三棱柱网格单元数为 35 。为了验证程序可靠性, 共 计算了 2 个不同流量条件下进气道性能, 对应进出 口气流压力比分别为 0.95078 和 0.74763 , 来流马 赫数 $M$ 为 0.21 , 攻角 $\alpha$ 为 $0^{\circ}$, 雷诺数为 $0.3885 \times 10^{6}$ 。

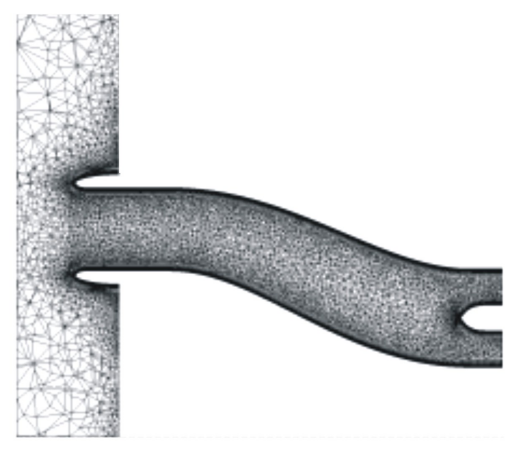

图 1 M2129 进气道模型网格分布

图 2 分别给出了采用 2 种不同湍流模型计算得 到的进气道上下壁面压力分布与试验值的对比情
况。可以看出,在不同流量条件下, SA 和 SST 湍流 模型计算得到的下表面分离位置较试验比均略靠 前, 但整体来说, 计算与试验得到的压力分布均吻合 较好, 说明程序可以捕捉到大 $\mathrm{S}$ 弯进气道内部典型 流动特征。表 1 给出了采用不同湍流模型计算得到 的进气道出口的总压恢复系数与试验值及文献值的 对比情况, 可以看出, 本文计算得到的总压恢复系数 与试验及文献值吻合较好, 尤其是在小流量系数条 件下, 与试验值差别不到 $1 \%$, 说明本文采用的数值 计算方法可以用于大 $\mathrm{S}$ 弯进气道性能分析, 验证了 程序的可靠性。

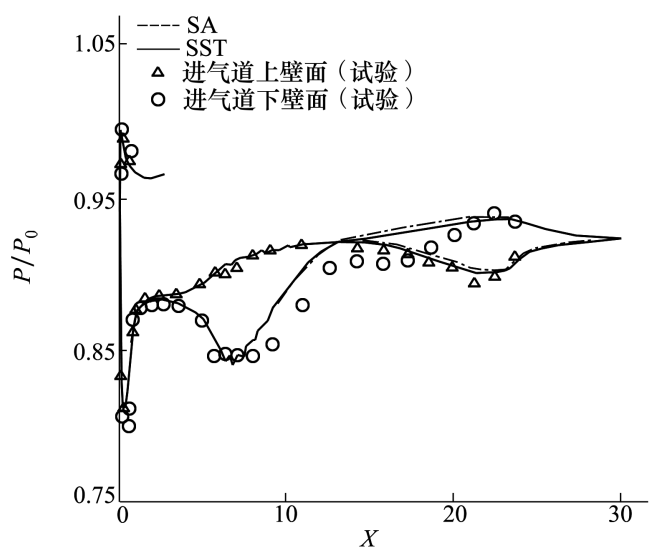

a) 小流量条件 $p / p_{b}=0.95078$

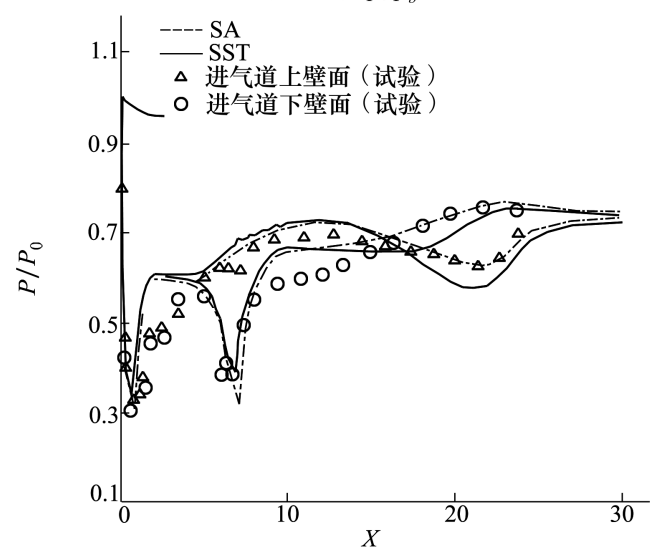

b) 大流量条件 $p / p_{b}=0.74763$

图 2 计算与试验进气道上下壁面压力对比

表 1 不同湍流模型进气道出口总压恢复系数对比

\begin{tabular}{ccc}
\hline 类型 & $\begin{array}{c}\text { 小流量系数 } \\
p / p_{b}=0.95078\end{array}$ & $\begin{array}{c}\text { 大流量系数 } \\
p / p_{b}=0.74763\end{array}$ \\
\hline $\mathrm{SA}$ & 0.98391 & 0.91054 \\
$\mathrm{SST}$ & 0.98397 & 0.90929 \\
文献值 & 0.98379 & 0.90794 \\
试验值 & 0.98974 & 0.92798 \\
\hline
\end{tabular}




\section{3 计算结果讨论分析}

本节利用前面介绍的数值计算方法, 对某带鼓 包的背负式大 $\mathrm{S}$ 弯进气道在超声速来流条件下的流 场特性及参数影响规律进行了一体化数值模拟研 究。该进气道内管道采用双大 $\mathrm{S}$ 弯曲设计, 唇口为 后掠形状, 无附面层隔道, 在进气口处采用鼓包设 计, 这个鼓包起到对来流的压缩作用, 并产生一个把 附面层气流推离进气道的压力分布。为了较好地对 流场特性进行捕捉, 数值模拟分析时采用了网格自 适应技术, 提升局部流场特性捕捉精度。图 3 给出 了自适应前后进气道人口附近网格分布。可以看 出, 网格自适应后进气道鼓包处的压缩激波模拟的 更加精细。

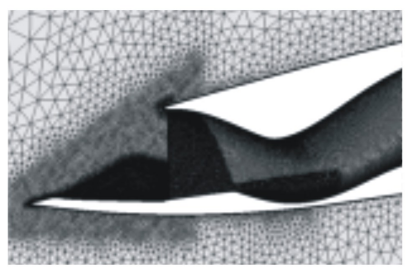

a) 自适应前

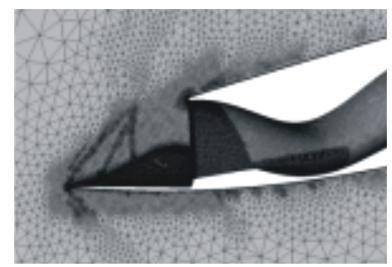

b）自适应后
图 3 背负式进气道自适应前后网格分布

\section{1 进气道超声速流场特性分析}

图 4 给出了该背负式进气道在超声速 $M$ 为 1.53 情况下计算得到的进气道总压恢复系数与试验值对 比曲线, 其中横坐标为流量系数, 纵坐标为总压恢复 系数, 可以看出, 计算得到的总压恢复系数与试验值 吻合较好, 随着流量系数的逐渐增大, 进气道出口总 压恢复逐渐降低, 总压损失严重。图 5 给出了不同 流量系数条件下出口截面上总压分布, 可以看出, 随 着流量系数的增大, 总压分布变得越来越不均匀。

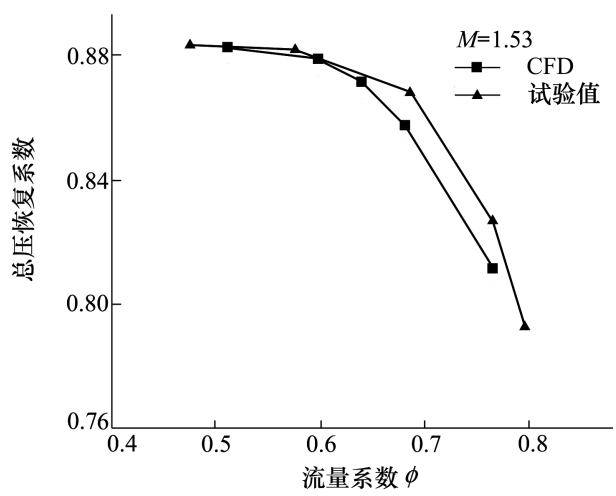

图 4 计算与试验总压恢复系数对比

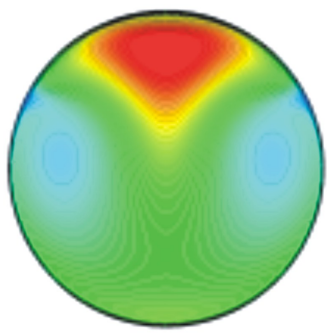

a) $M=1.53, \phi=0.6$

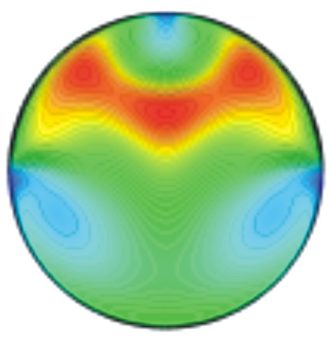

b) $M=1.53, \phi=0.77$
图 5 不同流量系数对应出口截面的总压分布

图 6 给出了在流量系数为 0.6 时进气道人口附 近马赫数分布云图, 可以看出, 机头产生第一道斜激 波后,鼓包压缩产生雉型波,系列锥形波经鼓包压缩 后形成末激波。在末激波后面, 气流变成亚声速, 进 人进气道内。在进气道外唇口附近, 在该流量系数 下进气道产生溢流, 使得唇口外表面气流加速, 局部 变成了超声速。随着进气道流量的增大, 进气口处 的末激波会逐渐发生内移, 造成总压损失逐渐加大, 进气道性能下降。

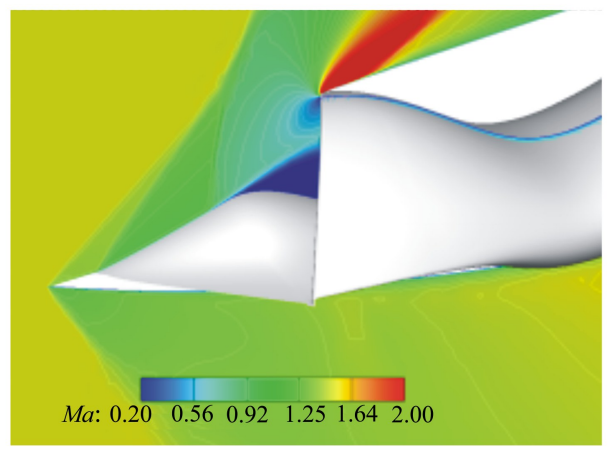

图 6 进气道人口附近马赫数分布云图

图 7 给出了进气道内不同截面的总压分布和空 间流线分布。可以看出, 气流在经过鼓包以后在进 气道的下壁面发生了分离,形成了一个较低动量的 区域,随着流动不断向下游传播发展。气流在 $\mathrm{S}$ 弯 管道内部, 由于在转弯位置曲壁顶部压强大于底部 压强, 在附面层内, 速度低于核心流, 为了平衡径向 压强梯度, 需要附面层内速度较低的气流向内转折 一个更大的角度, 从而出现由顶部到底部的横向流 动, 形成二次流。同时, 我们可以发现, 气流在经过 第二道 $\mathrm{S}$ 弯时, 由于曲壁产生的压力梯度改变符号, 使得下壁面附近的低能量区域有向顶部移动的趋 势。图 8 分别给出了流量系数在 0.6 和 0.77 情况下 进气道出口截面速度矢量分布, 图中云图采用马赫 
数着色。可以看出, 随着流量系数的不同, 出口截面 的二次流的形态会有所差别, 在小流量时, 呈现的是 一对反方向旋转的漩浴结构, 而在大流量时, 进气道 出口截面会出现 3 对涡的结构。

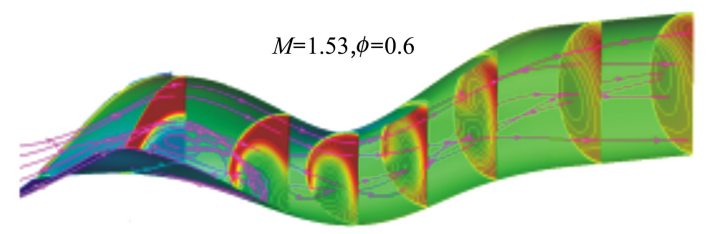

图 7 进气道内不同剖面总压及空间流线分布
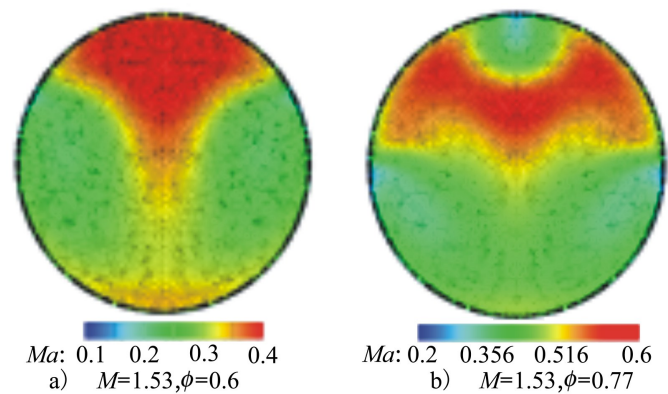

图 8 不同流量系数对应出口截面速度矢量图

\section{2 鼓包高度对进气道性能影响}

对于带鼓包的背负式大 $S$ 弯进气道, 影响其性 能的参数较多, 由于本文研究的进气道采用鼓包 + 后掠唇口的设计, 不同的鼓包几何参数会对进气道 的性能产生一定的影响, 本节主要考察进气道人口 处不同鼓包高度对进气道性能的影响。选取了 3 个 高度 $A 、 B 、 C$,具体外形示意图如图 9 所示。

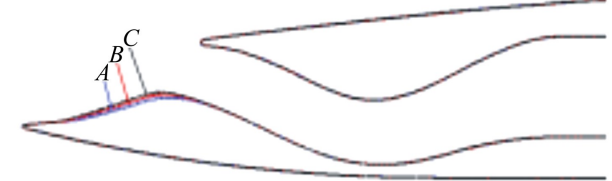

图 9 不同鼓包高度示意图

图 10 给出了不同鼓包高度对进气道性能影响 规律曲线, 可以看出, 随着鼓包高度的增大, 进气道 的总压恢复系数逐渐降低。为分析其原因,图 11 给 出了流量系数为 0.6 , 鼓包 $A$ 和鼓包 $C 2$ 种情况下进 气道人口处的激波分布。结果显示, 随着鼓包高度 的增加, 鼓包处的压力等值线发生后移, 在鼓包后面 越容易发生分离。图 12 给出了鼓包 $A$ 高度下进气 道内不同剖面的总压云图和空间流线分布, 与图 7 鼓包 $C$ 高度下进气道内不同剖面总压云图对比可
以看出,随着鼓包高度的升高, 鼓包后的低能量分离 区逐渐变大,在来流和 $\mathrm{S}$ 弯曲壁的逆压梯度共同作 用下逐渐向下游发展, 从而导致进气道性能逐渐下 降, 总压恢复系数逐渐降低。

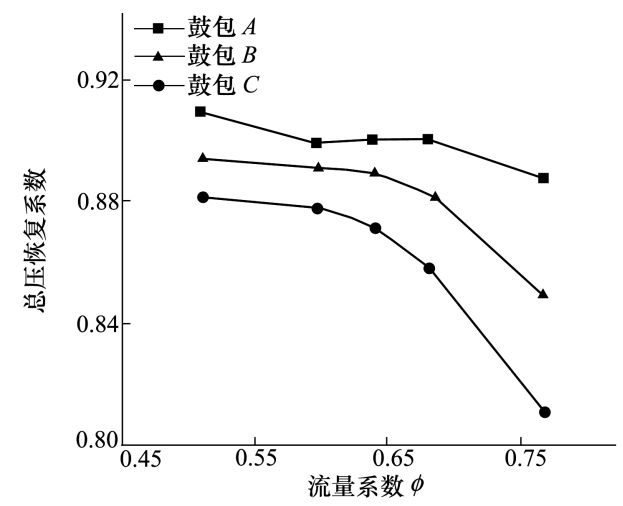

图 10 不同鼓包高度对进气道性能影响曲线

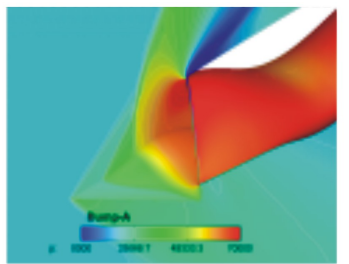

a) 鼓包 $A$

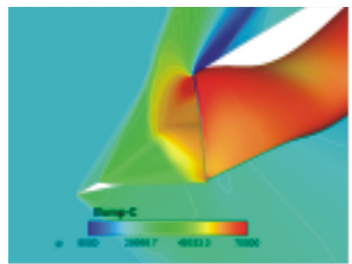

b）鼓包 $B$
图 11 不同鼓包高度对进气道人口附近波系影响

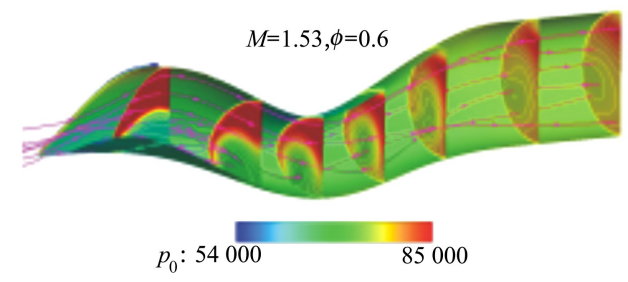

图 12 鼓包 $A$ 高度下进气道内总压和空间流线分布

\section{3 唇缘后掠角对进气道性能影响}

对于背负式进气道, 不同的外流条件会对进气 道人口处的气流产生较大的影响, 从而影响进气道 的性能。本节主要考察进气道不同唇缘后掠角对其 性能的影响, 我们选取了 3 个唇缘后掠角 $\left(35^{\circ}, 40^{\circ}\right.$, $\left.45^{\circ}\right)$ 进行了计算分析, 图 13 给出了不同唇缘后掠角 的外形对比示意图。图 14 给出了不同唇缘后掠角 对进气道性能影响的规律曲线, 可以看出, 随着后掠 角的增大, 进气道出口截面的总压恢复系数逐渐降 低, 为分析其原因, 图 15 给出了唇缘后掠角为 $35^{\circ}$ 和 $45^{\circ}$ 情况下的进气道对称面压力分布, 可以看出, 进气道唇口位于末激波后面, 处于亚声速区域, 随着 后掠角的增大, 进气道内的压力逐渐降低, 唇口位置 
产生的高压区位置逐渐后移, 从而影响了进气道的

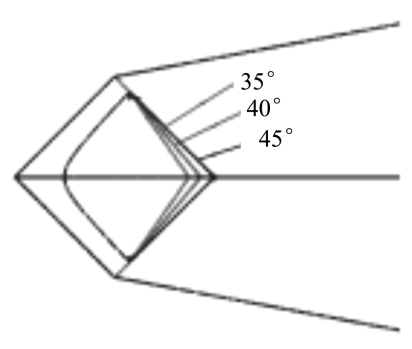

图 13 不同唇缘后掠角示意图

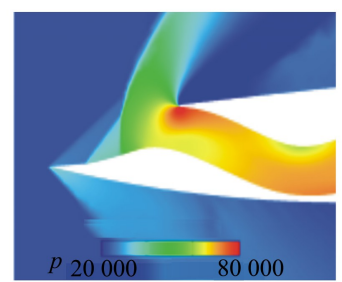

a) 后掠角 $35^{\circ}$

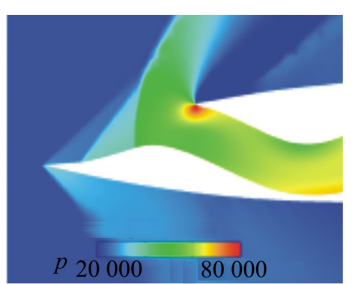

b) 后掠角 $45^{\circ}$
图 15 不同唇缘后掠角对称面压力分布云图对比

\section{4 结 论}

本文通过对带鼓包的背负式大 $\mathrm{S}$ 弯进气道流场 特性及参数影响规律的一体化数值模拟研究, 可以 得到以下结论：

1) 不同湍流模型对大 $S$ 弯进气道出口总压恢
人口气流, 导致进气道总压恢复系数逐渐降低。

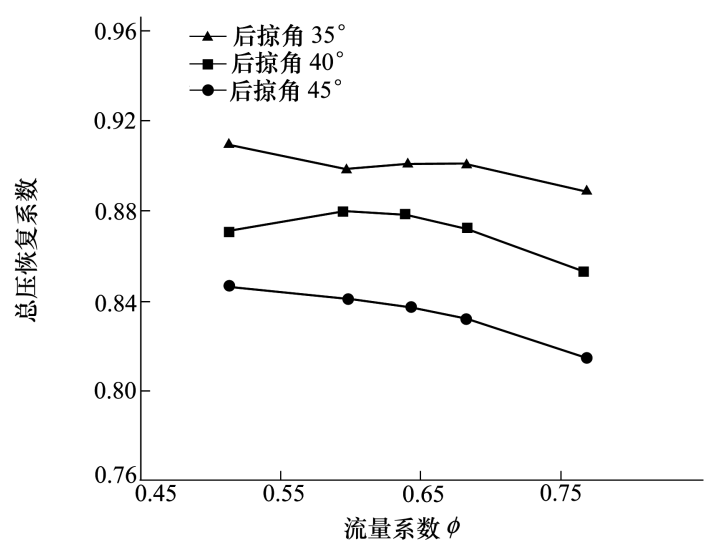

图 14 不同唇后掠角对进气道性能影响曲线

复系数预测影响较小, $\mathrm{SA}$ 和 SST 湍流模型计算得到 的 S 弯管道下表面分离位置较试验比均略靠前, 计 算得到的 $\mathrm{S}$ 弯管道内压力分布和出口总压恢复系数 均与试验值吻合较好, 说明本文采用的数值计算方 法可用于大 $\mathrm{S}$ 弯进气道内外流一体化性能分析。

2) 对于带鼓包的背负式大 S 弯进气道, 在超声 速时来流时, 进气道人口处鼓包压缩产生雉形波, 系 列锥形波不断压缩, 起到附面层排除的作用; 鼓包后 形成的低动量区域随着流动不断向下游发展, 在弯 曲管道曲率和压力梯度共同作用下, 产生二次流动, 流量系数不同,进气道出口截面的二次流形态差异 较大。

3) 分析了不同鼓包高度、唇缘后掠角对进气道 性能影响规律, 结果表明, 在一定范围内, 适当降低 鼓包高度或减小唇缘后掠角, 有利于进气道性能 改善。

\section{参考文献:}

[1] GRIDLEY M C, WALKER S H. Inlet and Nozzle Technology for 21st Century Fighter Aircraft[R]. ASME-1996-GT-244

[2] 石否, 郭荣伟. 蛇形进气道的电磁散射特性 [J]. 航空学报, 2007, 28(6)：1296-1310

SHI Lei, GUO Rongwei. Electromagnetic Scattering Characteristics of Serpentine Inlet[J]. Acta Aeronautica et Astronautica Sinica, 2007, 28(6):1296-1310 (in Chinese)

[3] 张乐, 周洲, 许晓平, 等. 飞翼无人机保形进排气系统动力数值模拟与流场特性分析 $[\mathrm{J}]$. 西北工业大学学报, 2015,33 (3) : 353-360

ZHANG Le, ZHOU Zhou, XU Xiaoping, et al. Numerical Simulation of Power and Flow Field Characteristics of Conformal Intake and Exhaust for Flying Wing Unmanned Aerial Vehicle[J]. Journal of Northwestern Polytechnical University, 2015, 33 (3) : 353-360 (in Chinese)

[4] 郁新华,刘斌, 陶于金, 等. 背负式进气道设计及其气动性能研究 $[\mathrm{J}]$. 西北工业大学学报, 2007, 25(2) : 270-273 
YU Xinhua, LIU Bin, TAO Yujin, et al. Top-Mounted Inlet Design and Its Aerodynamic Performance[ J]. Journal of Northwestern Polytechnical University, 2007, 25(2) : 270-273 (in Chinese)

[5] 陈颖秀,侯安平,张章,等. 前机身/进气道攻角特性的数值与试验研究 $[\mathrm{J}]$. 推进技术, 2014,35(6): 727-734

CHEN Yingxiu, HOU Anping, Zhang Zhang, et al. Numerical and Experimental Studies on Characteristics under Angle of Attack for Forebody-Inlet[J]. Journal of Propulsion Technology, 2014, 35(6) : 727-734 (in Chinese)

[6] WILliams T L, HUNT B L, SMELTZER D B, et al. Top-Mounted Inlet System Feeaibility for Transonic-Supersonic Fighter Aircraft Applications [R]. AGARD-CP-301

[7] RICHARD K S. An Overview of Military Aircraft Supersonic Inlet Aerodynamic[R]. AIAA-2012-0013

[8] HAMSTRA J W, SYLVESTER G T. System and Method for Diverting Boundaty Layer Air[P]. US, 5779189, 1998-07-14

[9] KIM S D. Aerodynamic Design of a Supersonic Inlet with a Parametric Bump[J]. Journal of Aircraft, 2009, 46( 1): 198-202

[10] TAN H J, GUO R W. Design and Wind Tunnel Study of a Top-Mounted Diverterless Inlet [J]. Chinese Journal of Aeronautics, $2004,17(2): 72-78$

[11] 张航,谭慧俊,李湘萍, 等. 类 X-47 狭缝式进气道的流动特征与工作性能 $[\mathrm{J}]$. 航空学报, 2009, 30(12):2243-2249

ZHANG Hang, TAN Huijun, LI Xiangping, et al. Flow Structure and Performance Characteristiscs of X-47-Like Slot-Shaped Inlet $[\mathrm{J}]$. Acta Aeronautica et Astronautica Sinica, 2009, 30(12) : 2243-2249 (in Chinese)

[12] 李大伟,张云飞,马东立. 背负式 $S$ 形进气道设计及数值分析 $[J]$. 推进技术, 2006, 27( 1):61-65

LI Dawei, ZHANG Yunfei, MA Dongli. Design and Numerical Investigation of Dorsal S-Shape Inet [J]. Journal of Propulsion Technology, 2006, 27(1): 61-65 (in Chinese)

[13] NEAL D D, DAN B. Inlet CFD Results: Comparison of Solver, Turbulence Model, Grid Density and Topology [R]. AIAA2013-3793

[14] CHAD M W, ZACH S D. Summary of the 3rd Propulsion Aerodynamics Workshop: S-duct Result[R]. AIAA-2017-4912

[15] MICHELLE L M, SCOTT A M, GISSEN A, et al. Inlet Flow Control and Prediction Technologies for Embedded Propulsion Systems $[\mathrm{R}]$. NASA/CR-2011-217237

[16] 梁德旺,李博. 无隔道进气道反设计及附面层排除机理分析 $[\mathrm{J}]$. 航空学报, 2005, 26( 3) :286-289

LIANG Dewang, LI Bo. Reverse Design of Diverterless Inlet and Mechanism of Diversion of Boundary Layer[J]. Acta Aeronautica et Astronautica Sinica, 2005, 26(3) : 286-289 (in Chinese)

[17] 宁乐,谭慧俊,孙姝. 有无边界层吸人对 S 弯进气道流动特性的影响 $[\mathrm{J}]$. 推进技术, 2017,38(2):266-274

NING Le, TAN Huijun, SUN Shu. Effects of Boundary Layer Ingestion on Flow Characteristics of an S-Shaped Inlet[ J]. Journal of Propulsion Technology, 2017, 38(2) : 266-274 (in Chinese)

[18] CHEN J T, ZHANG Y B, ZHOU N C, et al. Numerical Investigations of the High-Lift Configuration with M Flow Solver[J]. Journal of Aircraft, 2015, 52(4): 1051-1062

[19] GONG X Q, CHEN J T, ZHOU N C, et al. The Effects of Turbulence Model Correction on Drag Prediction of NASA Common Research Model[R]. AIAA-2014-3171

[20] LIU K W, ROSENDALE J V, VEEMEER W. 3D Shock Wave Visualization on Unstructured Grids [ C] // 1996 IEEE Symposium on Volume Visualization, San Francisco, California, 1996

[21] MOIGNE Y L. Adaptive Mesh Refinement Sensors for Vortex Flow Simulations [C] // Proceedings of the European Congress on Computational Methods in Applied Sciences and Engineering, 2004

[22] SPALART P R, ALLMARAS R. A One-Equation Turbulence Model for Aerodynamic Flows[R]. AIAA-1992-0439

[23] FLRIAN R Menter. Improved Two-Equation k- $\omega$ Turbulence Model for Aerodynamic Flow[ R]. NASA TM103975

[24] STANLEY R. WIND-US Flow Calculations for the M2129 S-Duct Using Structured and Unstructured Grids[R]. AIAA-2004-0525 


\title{
Numerical Investigation of Dorsal S-Shaped Inlet Flow Characteristic and Effects of Related Parameters
}

\author{
JIA Hongyin, ZHOU Guiyu, TANG Jing, WU Xiaojun, MA Mingsheng \\ (Computational Aerodynamics Institute, China Aerodynamics Research and Development Center, Mianyang 621000, China)
}

\begin{abstract}
The air intake design system of the dorsal intake combined with the S-shaped inlet has been widely used in various combat aircraft due to its good stealth characteristics. In this paper, the numerical simulation of the flow characteristic and influence law of various parameters for a typical air intake design with dorsal S-shaped inlet was carried out using the in-house large-scale parallel computational fluid dynamics (CFD) solver. Firstly, the numerical method was introduced and the solver was preliminarily validated by the well-known RAE M2192 inlet model. Then, the numerical calculation of the target air intake design was conducted and the distribution of shockwave at the entrance area, the second flow in the inlet and the flow at the exit section plane were analyzed in proper order. In addition, the influence of the bump height and lip sweep angles on the inlet performance was also studied. The simulation results show that the unique S-shaped design in the inlet will result in flow separation and secondary flow, ultimately causing total pressure loss, and different external geometry parameters have a great influence on the inlet performance. Within a certain range, proper reduction of the bump height or lip sweep angle can improve the inlet performance.
\end{abstract}

Keywords: dorsal air intake; S-shaped inlet; bump; total pressure recovery; second flow; related parameters; simulation; shockwave; CFD 